\title{
Validity of Serum miRNA 93 and miRNA 191 to Reduce Unnecessary Computed Tomography in Patients With Mild Head Trauma
}

\author{
Demet Tas ${ }^{\mathrm{a}}$, Onur Kaplana, Ozgur Sogut ${ }^{\mathrm{a}, \mathrm{b}}$
}

\begin{abstract}
Background: Indication for the appropriate use of cranial computed tomography (CCT) in patients with mild head trauma (MHT) based on history and physical examination alone remains unclear. Recent studies have been reported that $90 \%$ of patients with MHT who undergo CCT under the present clinical decision rules have no clinically important brain injuries. We aimed to investigate whether peripheral blood expression of microRNA 93 (miR93) and microRNA 191 (miR191) in patients with MHT can predict the presence or absence of intracranial injury, reducing the unnecessary use of CCT.
\end{abstract}

Methods: Fifty-nine consecutive adult patients with isolated MHT undergoing CCT based on the clinical decision guidelines of the New Orleans criteria and 91 age- and sex-matched controls were enrolled in this prospective observational cohort study. Patients were divided into two groups: those without or with traumatic intracerebral or extracerebral lesions identified by CCT. Patients were further divided into two subgroups based on the presence or absence of traumatic parenchymal lesions defined as traumatic brain injury (TBI).

Results: Mean serum miR93 and miR191 levels differed significantly between study groups. Of the 79 patients investigated, 16 exhibited trauma-relevant lesions on CCT scan $(\mathrm{CCT}+)$. With a cut-off limit of 0.15 , miR191 had an area under the curve value of 0.765 (0.640 - 0.889), with sensitivity of $68.1 \%$ and specificity of $68.8 \%$ in $\mathrm{CCT}+$ patients. Compared to MHT patients without TBI, mean serum miR191 levels were markedly elevated in patients with TBI. However, miR93 levels did not exhibit significant changes in either group.

Conclusions: Circulating miRNA levels increased after MHT and differentiated patients with and without intracranial or extracranial lesions demonstrable on CCT. Adding the measurement of serum miRNAs particularly miR191 to the clinical decision rules for a CCT scan

Manuscript submitted June 15, 2020, accepted June 26, 2020

Published online August 15, 2020

aDepartment of Emergency Medicine, Haseki Training and Research Hospital, University of Health Sciences, Istanbul, Turkey

${ }^{b}$ Corresponding Author: Ozgur Sogut, Department of Emergency Medicine, Haseki Training and Research Hospital, University of Health Sciences, Millet Street, Fatih, Istanbul 34096, Turkey. Email: ozgur.sogut@sbu.edu.tr

doi: https://doi.org/10.14740/jocmr4265 in patients with MHI could allow a reduction in scans.

Keywords: miRNA93; miRNA191; Mild head trauma; Traumatic brain injury; Cranial computed tomography

\section{Introduction}

Head trauma is one of the most important causes of mortality and morbidity in developed countries, especially in young populations $(<45$ years). It constitutes approximately $20 \%$ of patients admitted to hospital emergency departments (EDs) [1]. According to the literature, the most common causes of head trauma include traffic accidents, falls and blows to the head. Although severe head trauma is more frequently associated with disability and death, there are more frequent admissions to hospitals due to mild head trauma (MHT) $[2,3]$.

The New Orleans Head Trauma Computed Tomography (CT) guidelines and the Nexus II rule are commonly used to determine the indications for CT scan of the head in patients with MHT [4]. The New Orleans rule states that a cranial computed tomography (CCT) is necessary for patients with any of the following: headache, vomiting, age $>60$ years, alcohol or drug intoxication, persistent anterograde amnesia, presence of trauma above the clavicle, or seizure in MHT patients [5]. Indication for the appropriate use of CCT in patients with MHT based on history and physical examination alone remains unclear [6]. Some studies argue that MHT patients with a normal physical examination can be safely discharged from hospital, adding that having routine CT scans of the head in every cranial trauma patient would significantly increase healthcare costs $[7,8]$. These reports led to the investigation of several biochemical parameters in the literature with the aims of identifying indicators to determine the severity of trauma and prognosis in head trauma patients, and the indication to carry out a CT scan of the head as alternatives to trauma scoring $[9,10]$.

miRNAs are a post-transcriptional non-coding class of RNAs that can act on target proteins in neuronal development and are involved in intracellular signal transduction [10,11]. They are associated with several diseases varying from hyperlipidemia to cancer [11-13]. Molecular and cellular studies in recent years have shown that miRNAs play important roles as biomarkers in the pathogenesis and progression of traumatic 
brain injury (TBI). Serum concentrations of various specific microRNAs provide useful information regarding the diagnosis, severity and prognosis of TBI [10]. Although a large number of studies have investigated miRNA levels in experimentally induced TBI in rats [14-16], studies investigating serum miRNA levels in head injury in humans and their association with the severity of trauma and prognosis in the early stages after the trauma (within the first $24 \mathrm{~h}$ ) are limited $[17,18]$.

Therefore, the present study aimed to: 1) quantify the levels of microRNA 93 (miR93) and microRNA 191 (miR191) present in the sera of adult patients with MHT; 2) identify the relationships between TBI and the initial levels of miR93 and miR191 in these patients, and 3) determine the predictive role of these miRNAs in estimating intracranial or extracranial injuries in order to reduce the use of unnecessary CCT scans. We also discuss whether miR93 and miR191 levels are effective biomarkers for making appropriate decisions for obtaining CT scans of the head in patients with MHT.

\section{Materials and Methods}

\section{Study design and setting}

This prospective, observational cohort study was conducted in accordance with the 1989 Declaration of Helsinki and was approved by the Ethics Committee of Istanbul Haseki Training and Research Hospital (Institutional Review Board no. 515; date June 21, 2017). The present study was funded by the Istanbul Health Sciences University Board of Scientific Research Projects (funding no. 2018/006).

\section{Selection of participants}

Fifty-nine consecutive adult patients who were admitted to our tertiary care university hospital ED between June 2017 and December 2018 with isolated MHT (Glasgow coma scale (GCS) score $>13$ ) undergoing CCT based on the clinical decision rule of the New Orleans criteria, and 91 age- and sex-matched controls who exhibited no evidence of disease or acute trauma were enrolled in this prospective study.

Inclusion criteria were adult patients ( $\geq 18$ years of age) with MHT. During the first $24 \mathrm{~h}$ after admission to the ED, CCT was performed in patients with a GCS score of 14 or 15 and having at least one of the New Orleans rules (age 60 years or above, headache, vomiting, alcohol or drug intoxication, short-term memory deficits, signs of trauma above the clavicle and seizures). After vital functions had been monitored, written informed consent was obtained directly from the patient or from their authorized representative. Healthy volunteers were informed about the study protocol, and written consent was obtained from all participants prior to their participation in the study.

Patients were divided into two groups as follows: those without or with traumatic intracranial or extracranial lesions (e.g., skull fracture, brain swelling, cerebral contusion and intracerebral hematoma) shown on CCT. Patients were further divided into two subgroups based on the presence or absence of traumatic parenchymal lesions defined as TBI.

The serum levels of miR93 and miR191 were assessed in MHT patients and healthy controls using quantitative realtime polymerase chain reaction (RT-qPCR), where it is now accepted as the gold-standard method for detection of very small quantities of miRNAs [19]. For blood sampling, miRNA isolation and quantification, the examiners were blinded to the history, imaging result and diagnosis of the patients.

\section{Blood sampling}

Two venous blood samples of $5 \mathrm{~mL}$ each were drawn from the antecubital vein from patients and controls at the time of admission to the ED without the use of medications or serum infusions and collected in vacuum gel tubes. Plasma was separated by centrifugation at $2,515 \times g$ for 10 min using a centrifuge (Electro-mag M615E, Istanbul, Turkey) and immediately stored at $-80{ }^{\circ} \mathrm{C}$ until the assessment of miR93 and miR191 levels. Quantification was performed after serum samples reached room temperature.

\section{RNA isolation in serum samples}

Total RNA in serum samples was isolated using the Direct-zol RNA MiniPrep Kit according to the procedures specified in the manufacturer's protocol (Zymo Research, Irvine, CA, USA). An equal volume of room temperature $2 \times$ denaturing Trizol reagent was added to $700 \mu \mathrm{L}$ of a 32 Units-volume sample and immediately vortexed. The vortexed samples were then centrifuged at $12,000 \mathrm{rpm}$ for $10 \mathrm{~min}$ at $4{ }^{\circ} \mathrm{C}$ to separate the organic phase. The upper clear phase was transferred to a new tube. Next, room temperature $100 \%$ ethanol, at a volume 1.25 times the sample volume, was added to the tube and mixed well. This mixture (ethanol/lysate) was then filtered. The filter was washed with $700 \mu \mathrm{L}$ of miRNA Wash 1 Solution. This procedure was repeated twice with $2 / 3$ solution. Finally, RNA was collected from the filter in $50 \mu \mathrm{L}$ RNAase/DNAase-free water. Concentration and quality assessments of the obtained RNA were performed using a spectrophotometer (Thermo Scientific Multiskan GO, Vantaa, Finland). The samples were considered adequate if their A260/A280 ratio was in the range between 1.8 and 2.0. The RNA isolation kit (Direct-zol RNA MiniPrep Kit, Zymo Research) was used in compliance with the manufacturer's instructions for isolating total RNA from isolated and stored cardiomyocytes.

\section{RNA quantification by spectrophotometry}

Total RNA was quantified using a NanoDrop spectrophotometer (Thermo Fisher Scientific, Waltham, MA, USA). Samples with a concentration between 200 and $700 \mathrm{ng} / \mu \mathrm{L}$ were used for microarray analysis and RT-qPCR. Measurement of miRNAs by RT-qPCR is very specific and sensitive gold-standard method, allowing the detection of nucleic acids from a number 
of origins [20]. When preceded by reverse-transcription PCR, RT-qPCR is a powerful tool to measure miRNAs expression and provides careful validation of reference genes for miRNAs to obtain accurate miRNA data $[19,20]$.

\section{cDNA synthesis from RNA and miRNA synthesis from cDNA}

cDNA was synthesized using $1 \mu \mathrm{g}$ of the obtained total RNA in accordance with the information in the literature and with the manufacturer's protocol (Qiagen miScript Reverse Transcription Kit, Qiagen, Venlo, Netherlands) [10, 18, 21]. In brief, 3.5 $\mu \mathrm{L}$ RNA was mixed with the solution provided in the kit containing poly-A polymerase and reverse transcriptase to reach a final volume of $20 \mu \mathrm{L}$. cDNA was synthesized from total RNA after the resulting solution was incubated at $37^{\circ} \mathrm{C}$ for $60 \mathrm{~min}$ and then at $95{ }^{\circ} \mathrm{C}$ for $5 \mathrm{~min}$.

cDNA samples $(3 \mu \mathrm{L})$ were mixed with SYBR dye, universal primer, the miRNA-specific primer provided in the kit, and water to reach a final volume of $20 \mu \mathrm{L}$, and the mixture was loaded into the real-time PCR instrument. Fluorescence measurements were recorded during the lag phase of the reaction. Primers specific to miR93 and miR191 were used in the reactions along with the U6 primer as the reference gene. The crude miRNA data obtained by real-time PCR were analyzed using the delta delta $\mathrm{CT}(\Delta \Delta \mathrm{CT})$ method [18].

\section{Interpretation of miRNA levels}

miRNA levels were calculated using $\Delta$ ct values. When the nominal miRNA levels were less than the clinical outcomes, they corresponded to upregulation, and higher levels corresponded to downregulation. The result was determined on the basis of fold-change; the absolute value is of importance. As a negative nominal value results from the relative expression of miRNA rather than a reduction, it corresponds to upregulation, meaning that an increase occurred compared to normal levels.

$$
\begin{gathered}
\Delta \mathrm{ct}=\text { target gene mean ct }- \text { control gene mean ct } \\
\text { fold-change }=2^{-\Delta \mathrm{ct}}
\end{gathered}
$$

When calculating the fold-change value, a negative value is obtained as a constant, which prevents the miRNA value from being negative, indicating that a smaller numerical value will, in fact, correspond to upregulation (i.e., an increase).

\section{Statistical analysis}

The required sample size was calculated by power analysis before data collection based on information from previous studies $[17,18,21,22]$. It was estimated that at least 58 participants and 58 controls would be required to detect significant differences among the patient groups with a power of $95 \%$ and an alpha error of $5 \%$. The data collected in the study were analyzed using SPSS 15.0 for Windows (IBM Corp., Armonk, NY, USA). Descriptive statistics are expressed in numbers and percentages for categorical variables. Numerical variables are expressed as the mean, standard deviation and minimum and maximum values. Intergroup comparisons (controls vs. patients) were conducted using Chi-squared and Student's independent $t$-tests for normally distributed data (e.g., gender and age) and the Mann-Whitney U test for non-normally distributed data (e.g., miR93 and miR191 levels). The numerical variables in the patient groups were compared using MannWhitney U test (comparison of miR93 and miR191 levels) when the data conformed to non-normal distribution.

The ratios in the groups were compared using the Chisquared test. The decisive factor was analyzed using the logistic regression analysis forward method to determine the cut-off value for miR191. The significance level was set at $\mathrm{P}<0.05$.

\section{Results}

The mean age of the 79 patients with MHT was $41.10 \pm 17.70$ years (range 18 - 89 years), and 59 (75\%) were males. The mean age of the 92 healthy volunteers was $36.5 \pm 10.7$ years (range 20 - 65 years) and 75 (81\%) were males. There was no significant difference between the patients and the controls in terms of age $(\mathrm{P}=0.344)$ or gender $(\mathrm{P}=0.279)$.

The mean serum miR93 level in the control group was $2.85 \pm 2.26$ and the minimum and maximum values were -1.91 and 9.08, respectively. In the patient group, the mean serum miR93 level was $0.74 \pm 1.97$, and the minimum and maximum values were -4.67 and 4.53 , respectively. In the control group, the mean serum miR191 level was $3.07 \pm 2.72$ and the minimum and maximum levels were -3.69 and 9.30, respectively. In the patient group, the mean serum miR191 level was 0.48 \pm 1.70 and the minimum and maximum values were -3.31 and 3.46, respectively (Table 1 and Fig. 1). Mean serum miR93 and miR191 levels were significantly elevated in patients compared to the controls (both $\mathrm{P}<0.001$; Table 1 and Fig. 1). The fold-change value was determined by taking the absolute $\Delta \mathrm{ct}$ values of the mean serum miR93 and miR191 levels in the head trauma and control groups. Based on the fold-change values, the mean levels of miR93 and miR191 were 4.3- and 6.2fold higher, respectively, in head trauma patients compared to those in the control group (Fig. 2).

The mechanisms of injury in decreasing order of frequency in patients with MHT were falling from heights $(\mathrm{n}=50$, $63.3 \%)$, assault $(\mathrm{n}=23,29.1 \%)$, motor vehicle accident $(\mathrm{n}=$ 4, 5\%) and motor vehicle-pedestrian accident $(n=2,2.5 \%)$. Falling from heights was the most common mechanism for MHT. TBI, including epidural hematoma, subdural hematoma, subarachnoid hemorrhage, cerebral contusion and pneumocephalus, was present in $12(15.2 \%)$ patients with MHT. TBI was not detected in $67(84.8 \%)$ patients with MHT. The head CT scans revealed that $16(20.3 \%)$ patients had abnormal findings, including linear fractures, collapse fractures, or TBI, and $63(79.7 \%)$ patients had normal findings. Of these patients, $15(19 \%)$ received inpatient treatment and $64(81 \%)$ were discharged from the ED. In patients with abnormal CT scans of the head, the mean miR93 and miR191 levels were upregulated and significantly higher than those in patients with 
Table 1. Comparison of Age, Gender and Serum Quantities of miR93 and miR191 Between the Patient and Control Groups

\begin{tabular}{|c|c|c|c|c|c|}
\hline & & group & & group & P* \\
\hline & Mean \pm SD & Min. - Max. & Mean \pm SD & Min. - Max. & \\
\hline Age & $41.10 \pm 17.70$ & $18-89$ & $36.50 \pm 10.70$ & $20-65$ & 0.344 \\
\hline Male & $59(81.5 \%)$ & & $75(74.7 \%)$ & & \\
\hline Female & $20(18.5 \%)$ & & $17(25.3 \%)$ & & \\
\hline
\end{tabular}

Data are expressed in numbers, percentages, mean \pm standard deviation (SD), and minimum and maximum values. * $S$ tudent's $t$-test was used to compare age groups, and Chi-squared test was used to compare the gender distribution between groups. The Mann-Whitney $U$ test was used to compare the serum miRNA-93 and -191 levels between groups.

normal CCT findings $(\mathrm{P}=0.017$ and $\mathrm{P}=0.001$, respectively $)$ (Table 2 and Fig. 3).

miR93 and miR191 were examined using the logistic regression analysis forward method in a model to determine whether these parameters could predict abnormal CT findings in patients with MHT. Receiver operating characteristic (ROC) analysis identified a cut-off serum miR191 level of 0.15 for distinguishing the patients with abnormal CCT findings from normal, with $68.1 \%$ sensitivity and $68.8 \%$ specificity (area under the curve (AUC): $0.765,95 \%$ confidence interval (CI): $0.640-0.889, \mathrm{P}=0.016$; Fig. 4). Serum miR191 levels of 0.15 and above predicted the normal findings in the CT scan in patients with MHT, whereas values below 0.15 indicated that abnormal findings in the CT scan were present. A significant

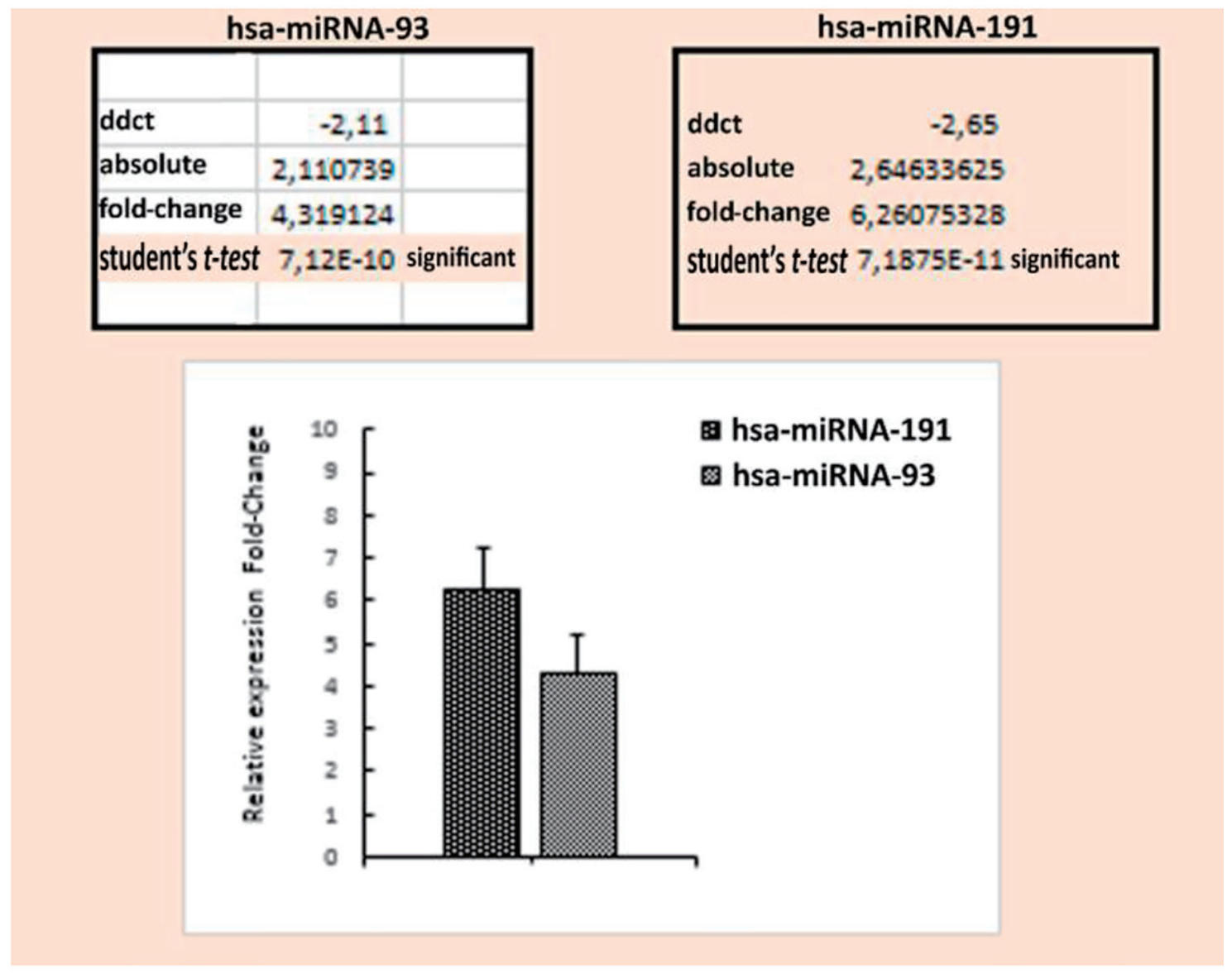

Figure 1. Comparison of serum miR93 and miR191 fold-change levels between the patient and control groups. Data are presented as numbers, percentages and mean \pm standard deviation (SD). The Mann-Whitney $U$ test was performed to compare miR93 and miR191 levels. 


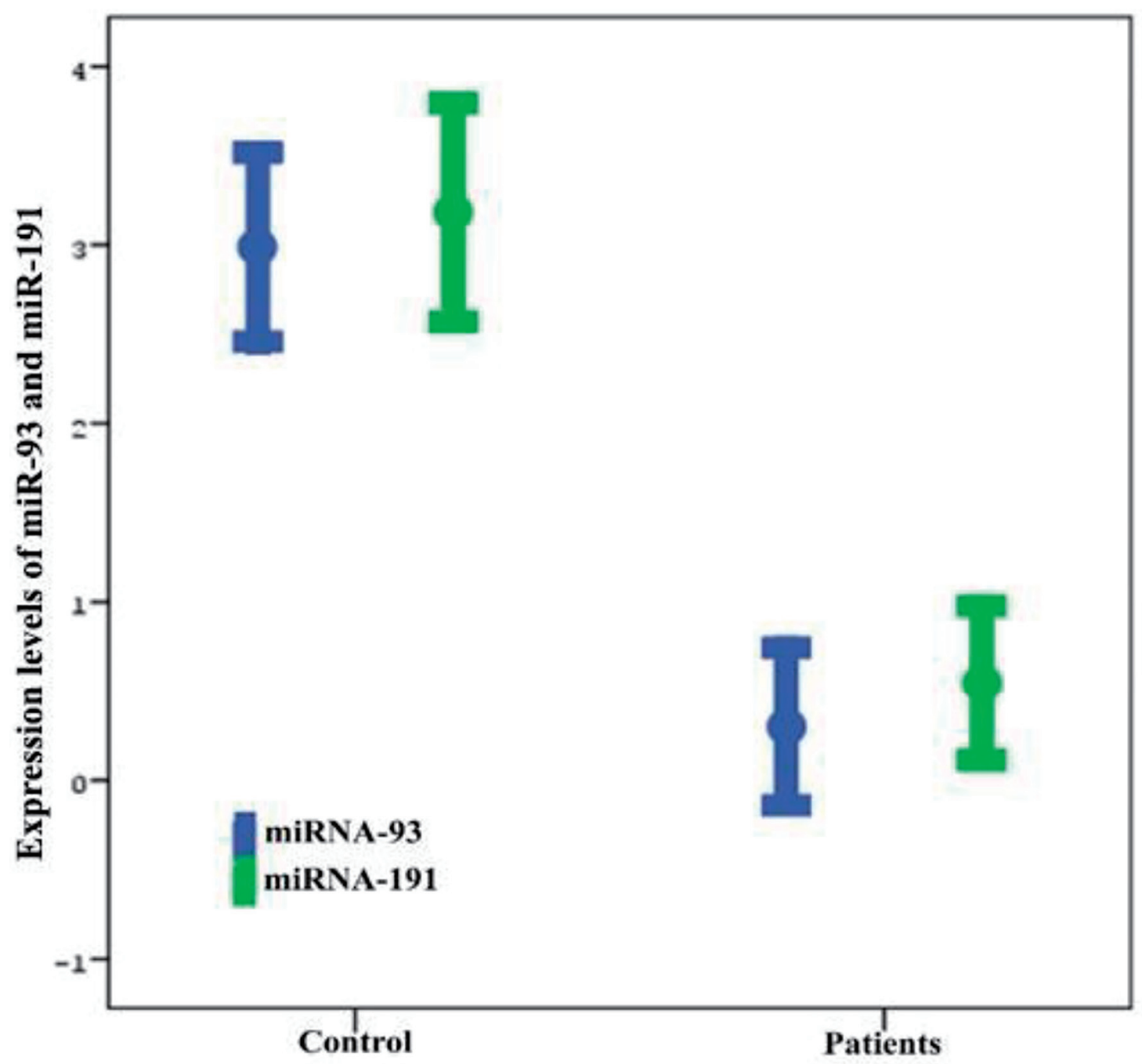

Figure 2. Serum miR93 and miR191 levels in the patient and control groups.

cut-off value for the miR93 level to predict the presence of pathological findings in CCT scans was not found in patients with mild head injury (AUC: 0.680, CI: $0.545-0.816, \mathrm{P}=$ $0.053)$.

The mean serum miR191 levels were significantly higher in MHT patients with TBI than in those without TBI $(\mathrm{P}=$
0.017; Table 3). However, the mean levels of serum miR93 were not significantly different between groups $(\mathrm{P}=0.145$; Table 3). In the ROC analysis, significant efficacy of the serum miR191 cut-off value of 0.069 was observed in distinguishing the patients with TBI from patients without TBI (AUC: 0.712, 95\% CI: 0.563 - 0.862, P=0.032; Fig. 5). Serum miR191 lev-

Table 2. Comparison of Serum miR93 and miR191 Levels Based on Findings of Cranial Computed Tomography (CCT) Scans

\begin{tabular}{|c|c|c|c|c|c|c|c|}
\hline & \multicolumn{6}{|c|}{ Findings in head CT scan } & \multirow{3}{*}{$P^{*}$} \\
\hline & \multicolumn{3}{|c|}{ Normal } & \multicolumn{3}{|c|}{ Abnormal } & \\
\hline & Mean \pm SD & Min. - Max. & Median & Mean \pm SD & Min. - Max. & Median & \\
\hline $\operatorname{miR} 191$ & $0.89 \pm 1.57$ & $-2.63-3.46$ & 1.08 & $-0.71 \pm 1.55$ & $-3.31-1.51$ & -0.83 & 0.001 \\
\hline
\end{tabular}

Data are presented as numbers, percentages, mean \pm standard deviation (SD) and minimum and maximum values. *The Mann-Whitney $U$ test was used to compare serum miR93 and miR191 levels between groups. Normal CT scan findings, including patients in the absence of traumatic intracranial or extracranial lesions (e.g., skull fracture, brain swelling, cerebral contusion and intracerebral hematoma). Abnormal CT scan findings, including linear fractures, collapse fractures, or traumatic brain injury (TBI). TBI, traumatic parenchymal lesions including epidural hematoma, subdural hematoma, subarachnoid hemorrhage, cerebral contusion and pneumocephalus. 


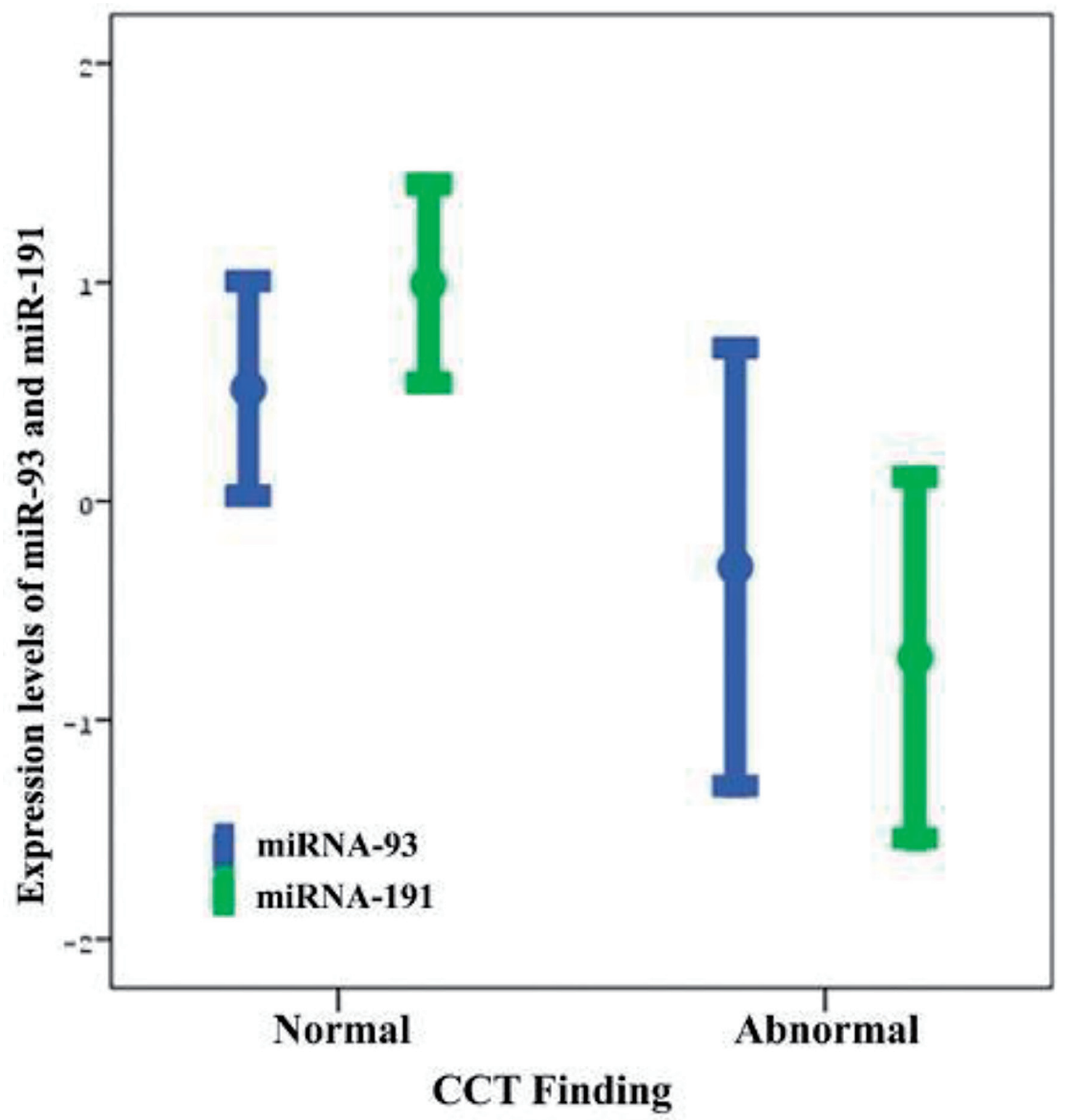

Figure 3. Distribution of serum miR93 and miR191 levels by cranial computed tomography (CCT) scan findings.

els of 0.069 and above predicted the absence of TBI in patients with MHT, whereas values below 0.069 indicated that TBI was present. Therefore, the cut-off value for the presence of TBI was determined to be $<0.069(66.7 \%$ sensitivity and $58.3 \%$ specificity; Fig. 5).

However, a significant cut-off value for the serum miR93 level for determining the presence of TBI was not found in head trauma patients (AUC: $0.660, \mathrm{CI}: 0.522-0.798, \mathrm{P}=$ 0.260).

\section{Discussion}

The present study was the first clinical trial to investigate whether serum levels of miR93 and miR191 in patients with
MHT can predict the presence or absence of intracranial injury for reducing the use of unnecessary CCT during the early post-traumatic period (within the first $24 \mathrm{~h}$ ). Thus, this work revealed the expression of miR93 and miR191 in the sera of patients with MHT, and found that miR191, in particular, may ameliorate the indication for the need for initial CCT scans.

MHT is defined as a clinical condition in which the patient has a GCS score of 14 or 15 , suffers a loss of consciousness for a duration shorter than $30 \mathrm{~min}$ and has a duration of hospital stay of less than $48 \mathrm{~h}[2,8]$. Today, MHT accounts for $>80 \%$ of patients admitted to EDs for the treatment of head trauma. Intracranial pathologies are detected in a very small number of these patients. Due to the high numbers of these patients, carrying out head CT scans in every patient poses serious financial burdens $[7,8]$. 


\section{ROC Curve (miR-191)}

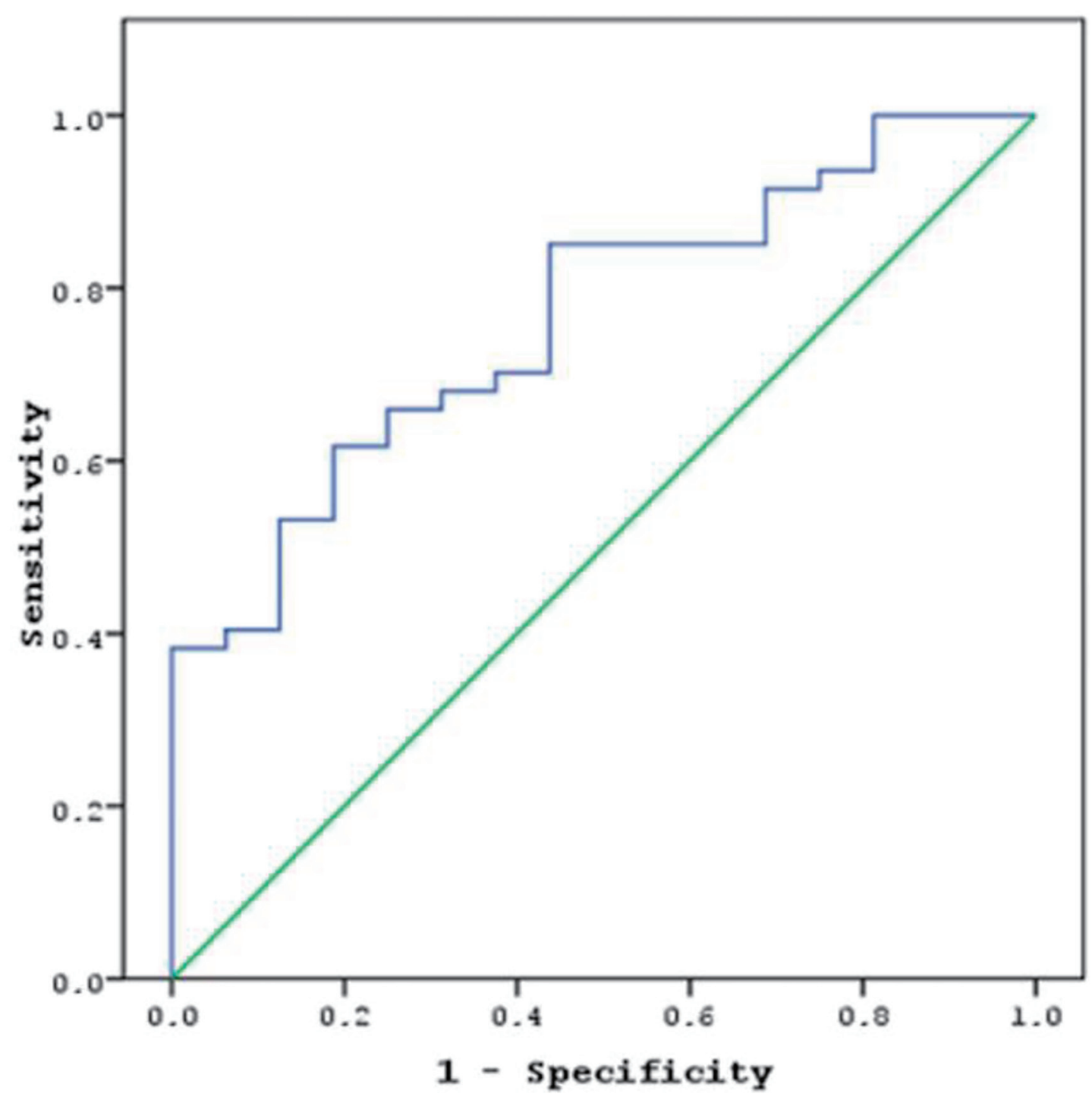

Figure 4. The sensitivity and specificity of miR191 levels in predicting the abnormal findings in the cranial computed tomography $(C C T)$ scan (area under the curve (AUC): 0.765; confidence interval $(\mathrm{Cl}): 0.640-0.889 ; \mathrm{P}=0.016$ ).

Controversy exists over whether a decision to carry out a head CT scan in patients with MHT can be made based on medical history and physical examination alone. Therefore, several clinical decision-making criteria have been developed to reduce the number of CT scans and the rates of radiation exposure [6]. Canadian, New Orleans and Nexus II are among the

Table 3. Comparison of Serum miR93 and miR191 Levels Between Patients With and Without Traumatic Brain Injury

\begin{tabular}{|c|c|c|c|c|c|c|c|}
\hline & \multicolumn{6}{|c|}{ Traumatic brain injury } & \multirow{3}{*}{$P *$} \\
\hline & \multicolumn{3}{|c|}{ Yes } & \multicolumn{3}{|c|}{ No } & \\
\hline & Mean \pm SD & Min. - Max. & Median & Mean \pm SD & Min. - Max. & Median & \\
\hline $\operatorname{miR} 191$ & $-0.56 \pm 1.63$ & $-3.31-1.51$ & -0.42 & $0.72 \pm 1.64$ & $-3.12-3.46$ & 1.01 & 0.017 \\
\hline
\end{tabular}

Data are expressed in numbers, percentages, mean \pm standard deviation (SD) and minimum and maximum values. The Mann-Whitney $U$ test was used to compare serum miR93 and miR191 levels between groups. 


\section{ROC Curve (miR-191)}

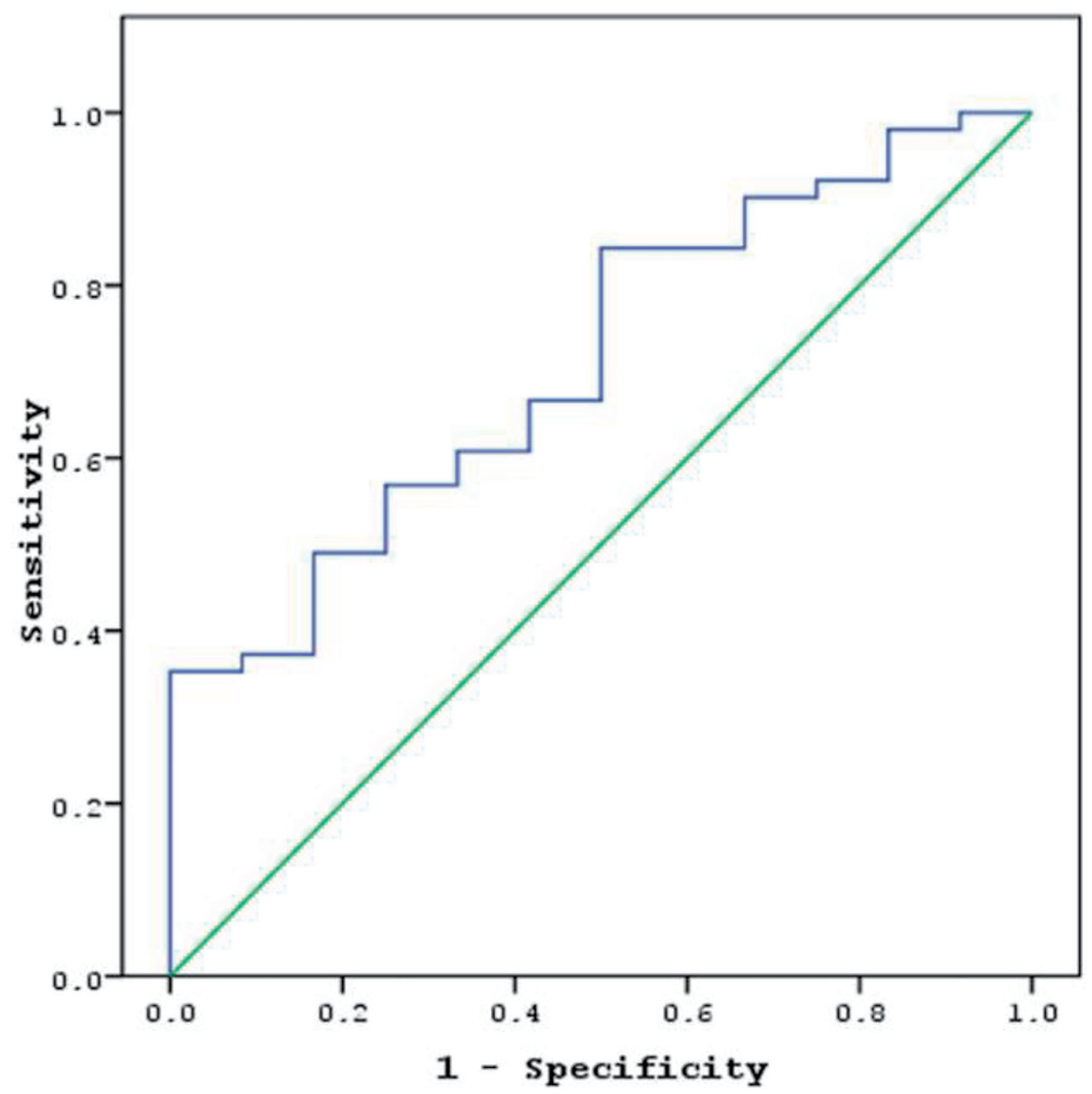

Figure 5. The sensitivity and specificity of serum miR191 levels in determining the presence of traumatic brain injury in patients with MHT (area under the curve (AUC): 0.712; confidence interval $(\mathrm{Cl})$ : $0.563-0.862 ; \mathrm{P}=0.032$ ).

most commonly used clinical decision-making rules for CCT in adults with MHT $[4,5]$. A routine CT scan of the head is not recommended when a patient with MHT does not meet any of the New Orleans or Nexus II criteria [5]. Recent clinical studies have reported that $90 \%$ of MHT patients undergoing head CT scans had no clinically significant brain injury according to current clinical decision-making rules [23]. Likewise, in the present study, 63 (79.7\%) patients had normal CCT findings based on New Orleans clinical decision-making criteria.

miRNAs are important post-transcriptional regulators of complementary mRNA targets, and have been implicated in the pathophysiology of acute brain injury [24, 25]. They have been suggested to be beneficial biomarkers for physiological and pathological processes because they are protected from RNase activity in the peripheral blood [26]. Numerous reference genes, such as small nuclear RNAs, have been commonly used for miRNA quantification [20]. These human miRNA molecules represent similar properties, such as RNA stability and size and are abundantly expressed. However, their expression levels can change under different experimental conditions and may be affected in various diseases [20, 27]. miR191 was the most consistently expressed miRNA across different human tissues, followed by miR93, miR106a, miR17-5p and miR25 [28].

Human and experimental studies have identified various specific miRNAs as biomarkers in serum (e.g., miR425-p, 
$-21,-93,-191,-499,-18 a,-203,-146 a,-149$ and $-23 b)$ and in cerebrospinal fluid (e.g., miR328, -362-3p, -451 and -486a) as possible indicators of the diagnosis, severity and prognosis of TBI [10, 16, 26, 29]. Several studies have reported that the miR93, miR191 and miR499 subtypes were upregulated in the cerebral cortex and dorsal hippocampus of rats that underwent experimentally induced TBI $[10,16,17,26]$. However, it is unclear whether these three miRNA subtypes are expressed in human serum after TBI. Therefore, in this study, we determined the expression levels of miR93 and miR191, and reported increased levels in the sera of MHT patients in experimental head trauma models, and evaluated the associations of these two miRNA subtypes with TBI using RT-qPCR.

The number of studies investigating an association between miRNA levels and the severity and prognosis of TBI in humans is very limited $[17,18,21]$. Yang et al [18] conducted a clinical study in 76 patients with TBI admitted to the inpatient service to be treated by either conservative or surgical methods and 38 controls. They quantified serum miR93, miR191 and miR499 levels in blood samples collected from patients during a period of 21 days. The study demonstrated that the serum levels of these three respective miRNA subtypes were significantly higher in patients than in the control group. In addition, the serum levels of these three miRNA subtypes were significantly elevated in patients with increased severity of trauma and with poor outcomes. In the limitations section of the study, the authors stated the serum levels of the miRNA subtypes were not obtained at the time of admission, and miRNA levels in the blood samples may have changed after treatment [18]. However, unlike the cited work, we quantified the serum levels of miR93 and miR191 at the time of admission to the ED before medical and surgical treatments to investigate their predictive values in determining pathological findings in emergency head CT and in indicating the presence of TBI. Among the miRNA subtypes examined in our study, we found a significant increase in the serum miR93 and miR191 levels in MHT patients within the first $24 \mathrm{~h}$ after trauma compared to those in the control group, consistent with the results of Yang et al. Our study also found that the mean serum levels of miR93 and miR191 were 4.3and 6.2-fold higher, respectively, in MHT patients than in the control group. Regarding CCT scan findings, the mean serum miR93 and miR191 levels were significantly higher and upregulated in patients with abnormal CCT scan findings compared to those in patients with normal CCT findings (CT). Serum miR191 levels of 0.15 and over were defined as the cut-off values for "CCT-" patients. On the other hand, levels below 0.15 were defined as the cut-off values for "CCT+" patients (68.1\% sensitivity and 68.8\% specificity; AUC: 0.765 ; CI: $0.640-0.889)$. These results suggest that serum miR93 and miR191 levels are upregulated in patients with MHT compared to those in control subjects. In our study, among the miRNA subtypes, the serum levels of miR191 were beneficial in decreasing the number of unnecessary CCT scans, reducing radiation exposure, and decreasing healthcare costs in MHT patients when used in combination with clinical decision-making rules.

In our study, when patients were evaluated in accordance with the presence of TBI, the mean serum levels of miR191 in TBI patients were significantly higher than those in patients without TBI. Serum miR191 levels of 0.069 and above predicted the absence of TBI in patients with MHT, and values below 0.069 were defined as cut-off values for the presence of TBI (66.7\% sensitivity and 58.3\% specificity; AUC: 0.712 ; CI: $0.563-0.862)$. Our findings indicated that the serum levels of miR191 may play a significant role in the pathogenesis of TBI in MHT patients and may also serve as a new biomarker for the diagnosis of TBI. This study is the first step toward validating thresholds for studies integrating miRNAs into clinical decision-making rules to detect TBI in patients with MHT. However, further large-scale randomized controlled studies are warranted to confirm this association and to recognize miRNA levels as biomarkers to be used in combination with clinical decision-making criteria in determining the indications for CCT scans.

There are some limitations to this study, the most important being the small sample size and single center design. In addition, a single measurement of serum miR93 and miR191 was taken within the first $24 \mathrm{~h}$ after MHT. Although most of the MHT patients $(79.7 \%)$ in the present study had normal CCT findings, and serum miR191 levels of 0.15 and above indicated the normal findings in the head CT scans with $68.1 \%$ sensitivity and $68.8 \%$ specificity, CCT can still be necessary to diagnosis severity or characteristics of trauma immediately in an ED setting. With repeated measurements at the followup MHT patients, changes in serum miRNAs levels could be observed in order to reduce the unnecessary use of CCT. Thus, the predictive role of these miRNAs in estimating intracranial or extracranial injuries deserves further study. These issues should be considered in future studies involving patients with MBT.

\section{Conclusions}

We found that serum miR191 and miR93 levels were upregulated and significantly higher in patients with MHT than in controls. Furthermore, these circulating serum miRNA levels increased after MHT, and can distinguish between those with and without intracranial or extracranial lesions demonstrable on CCT. miRNAs, particularly miR191 concentrations in patients with MHT, can provide additional information to improve indications for the need for an initial CCT scan. These results also suggest that serum miR191 levels play a significant role in the pathogenesis of TBI in patients with MHT. For this reason, we suggest that miR191 be used in combination with clinical decision-making rules in determining whether a CCT scan is required in patients with MHT.

\section{Acknowledgments}

This work was awarded as the best oral presentation by Scientific Committee of the Third International Family Medicine and Emergency Medicine Symposium that was held in Cyprus, November 29 - December 2, 2018. 


\section{Financial Disclosure}

This research was supported by Grant 2018/006 from University of Health Sciences Board of Scientific Research Projects, Istanbul, Turkey.

\section{Conflict of Interest}

None to declare.

\section{Informed Consent}

Informed consent was given by all study participants.

\section{Author Contributions}

Study concept and design: OS and DT. Patient recruitment: DT, OS, OK and TA. Acquisition of data: DT, OS and OK. Analysis and interpretation of data: DT and OS. Statistical analysis: OS and DT. Drafting of manuscript: DT and OS. Critical revision of the manuscript: OS, DT, OK and TA. Study supervision: OS.

\section{Data Availability}

The authors declare that data supporting the findings of this study are available within the article.

\section{References}

1. Olshaker JS, Whye DW, Jr. Head trauma. Emerg Med Clin North Am. 1993;11(1):165-186.

2. Jennett B. Epidemiology of head injury. J Neurol Neurosurg Psychiatry. 1996;60(4):362-369.

3. Sauaia A, Moore FA, Moore EE, Moser KS, Brennan R, Read RA, Pons PT. Epidemiology of trauma deaths: a reassessment. J Trauma. 1995;38(2):185-193.

4. Haydel MJ, Preston CA, Mills TJ, Luber S, Blaudeau E, DeBlieux PM. Indications for computed tomography in patients with minor head injury. N Engl J Med. 2000;343(2):100-105.

5. Papa L, Stiell IG, Clement CM, Pawlowicz A, Wolfram A, Braga C, Draviam S, et al. Performance of the Canadian CT Head Rule and the New Orleans Criteria for predicting any traumatic intracranial injury on computed tomography in a United States Level I trauma center. Acad Emerg Med. 2012;19(1):2-10.

6. Jagoda AS, Bazarian JJ, Bruns JJ, Jr., Cantrill SV, Gean $\mathrm{AD}$, Howard PK, Ghajar J, et al. Clinical policy: neuroimaging and decisionmaking in adult mild traumatic brain injury in the acute setting. Ann Emerg Med. 2008;52(6):714-748.

7. Jones LA, Morley EJ, Grant WD, Wojcik SM, Paolo
WF. Adherence to head computed tomography guidelines for mild traumatic brain injury. West J Emerg Med. 2014;15(4):459-464.

8. Boran BO, Barut N, Akgun C, Celikoglu E, Bozbuga M. [Indications for computed tomography in patients with mild head injuries]. Ulus Travma Acil Cerrahi Derg. 2005;11(3):218-224.

9. Kulbe JR, Geddes JW. Current status of fluid biomarkers in mild traumatic brain injury. Exp Neurol. 2016;275(Pt 3):334-352.

10. Martinez B, Peplow PV. MicroRNAs as diagnostic markers and therapeutic targets for traumatic brain injury. Neural Regen Res. 2017;12(11):1749-1761.

11. Mitchell PS, Parkin RK, Kroh EM, Fritz BR, Wyman SK, Pogosova-Agadjanyan EL, Peterson A, et al. Circulating microRNAs as stable blood-based markers for cancer detection. Proc Natl Acad Sci U S A. 2008;105(30):1051310518.

12. Selth LA, Tilley WD, Butler LM. Circulating microRNAs: macro-utility as markers of prostate cancer? Endocr Relat Cancer. 2012;19(4):R99-R113.

13. Yerlikaya FH, Can U, Alpaydin MS, Aribas A. The relationship between plasma microRNAs and serum trace elements levels in primary hyperlipidemia. Bratisl Lek Listy. 2019;120(5):344-348.

14. Redell JB, Liu Y, Dash PK. Traumatic brain injury alters expression of hippocampal microRNAs: potential regulators of multiple pathophysiological processes. J Neurosci Res. 2009;87(6):1435-1448.

15. Sabirzhanov B, Zhao Z, Stoica BA, Loane DJ, Wu J, Borroto C, Dorsey SG, et al. Downregulation of miR-23a and miR-27a following experimental traumatic brain injury induces neuronal cell death through activation of proapoptotic Bcl-2 proteins. J Neurosci. 2014;34(30):1005510071.

16. Liu L, Sun T, Liu Z, Chen X, Zhao L, Qu G, Li Q. Traumatic brain injury dysregulates microRNAs to modulate cell signaling in rat hippocampus. PLoS One. 2014;9(8):e103948.

17. Redell JB, Moore AN, Ward NH, 3rd, Hergenroeder GW, Dash PK. Human traumatic brain injury alters plasma microRNA levels. J Neurotrauma. 2010;27(12):2147-2156.

18. Yang T, Song J, Bu X, Wang C, Wu J, Cai J, Wan S, et al. Elevated serum miR-93, miR-191, and miR-499 are noninvasive biomarkers for the presence and progression of traumatic brain injury. J Neurochem. 2016;137(1):122129.

19. Schwarzenbach H, da Silva AM, Calin G, Pantel K. Data Normalization Strategies for MicroRNA Quantification. Clin Chem. 2015;61(11):1333-1342.

20. Meyer SU, Pfaffl MW, Ulbrich SE. Normalization strategies for microRNA profiling experiments: a 'normal' way to a hidden layer of complexity? Biotechnol Lett. 2010;32(12):1777-1788.

21. Di Pietro V, Ragusa M, Davies D, Su Z, Hazeldine J, Lazzarino G, Hill LJ, et al. MicroRNAs as Novel Biomarkers for the Diagnosis and Prognosis of Mild and Severe Traumatic Brain Injury. J Neurotrauma. 2017;34(11):19481956. 
22. Gosho M, Nagashima K, Sato Y. Study designs and statistical analyses for biomarker research. Sensors (Basel). 2012;12(7):8966-8986.

23. Bouvier D, Oddoze C, Ben Haim D, Moustafa F, Legrand A, Alazia M, Jehle E, et al. [Interest of S100B protein blood level determination for the management of patients with minor head trauma]. Ann Biol Clin (Paris). 2009;67(4):425-431.

24. Yang Z, Zhong L, Xian R, Yuan B. MicroRNA-223 regulates inflammation and brain injury via feedback to NLRP3 inflammasome after intracerebral hemorrhage. Mol Immunol. 2015;65(2):267-276.

25. Bache S, Rasmussen R, Rossing M, Hammer NR, Juhler M, Friis-Hansen L, Nielsen FC, et al. Detection and quantification of microRNA in cerebral microdialysate.
J Transl Med. 2015;13:149.

26. Sun W, Julie Li YS, Huang HD, Shyy JY, Chien S. microRNA: a master regulator of cellular processes for bioengineering systems. Annu Rev Biomed Eng. 2010;12:1-27.

27. Mahdipour M, Van Tol HT, Stout TA, Roelen BA. 225 validation of reference micrornas for normalizing expression data generated by quantitative pcr. Reproduction, fertility, and development. 2014;27:202.

28. Peltier HJ, Latham GJ. Normalization of microRNA expression levels in quantitative RT-PCR assays: identification of suitable reference RNA targets in normal and cancerous human solid tissues. RNA. 2008;14(5):844-852.

29. Redell JB, Zhao J, Dash PK. Altered expression of miRNA-21 and its targets in the hippocampus after traumatic brain injury. J Neurosci Res. 2011;89(2):212-221. 